\title{
Comparing $\mathrm{TiO}_{2}$ Nanoparticle Formulations: Stability and Photoreactivity Are Key Factors in Acute Toxicity to Daphnia magna
}

\author{
Jeffrey M. Farner, Rachel Cheong, Emeric Mahé, Hemanshu Anand, Nathalie Tufenkji* \\ Department of Chemical Engineering, McGill University, Montreal, Quebec, Canada \\ *Corresponding Author Phone: (514) 398-2999; Fax: (514) 398-6678; E-mail: nathalie.tufenkji@mcgill.ca
}

\section{Environmental Significance Statement}

Engineered titanium dioxide nanoparticles $\left(\mathrm{TiO}_{2} \mathrm{NPs}\right)$ are increasingly detected in the environment. Due to the many applications of $\mathrm{TiO}_{2}$, formulations of different particle size, morphology, and mineral form exist that may affect the stability and toxicity of the released NPs in complex systems. Variations in behavior and impact on aquatic species amongst $\mathrm{TiO}_{2}$ formulations remains poorly understood. This study compares two types of $\mathrm{TiO}_{2} \mathrm{NPs}$ in terms of photoreactivity and aggregation. The type of $\mathrm{TiO}_{2}$, the presence of UV light, and natural organic matter are all observed to impact the toxicity to Daphnia magna. This work highlights the importance of considering both the aquatic chemistry and the physicochemical characteristics of the $\mathrm{TiO}_{2} \mathrm{NPs}$ in determining environmental risk.

\section{TOC Art}

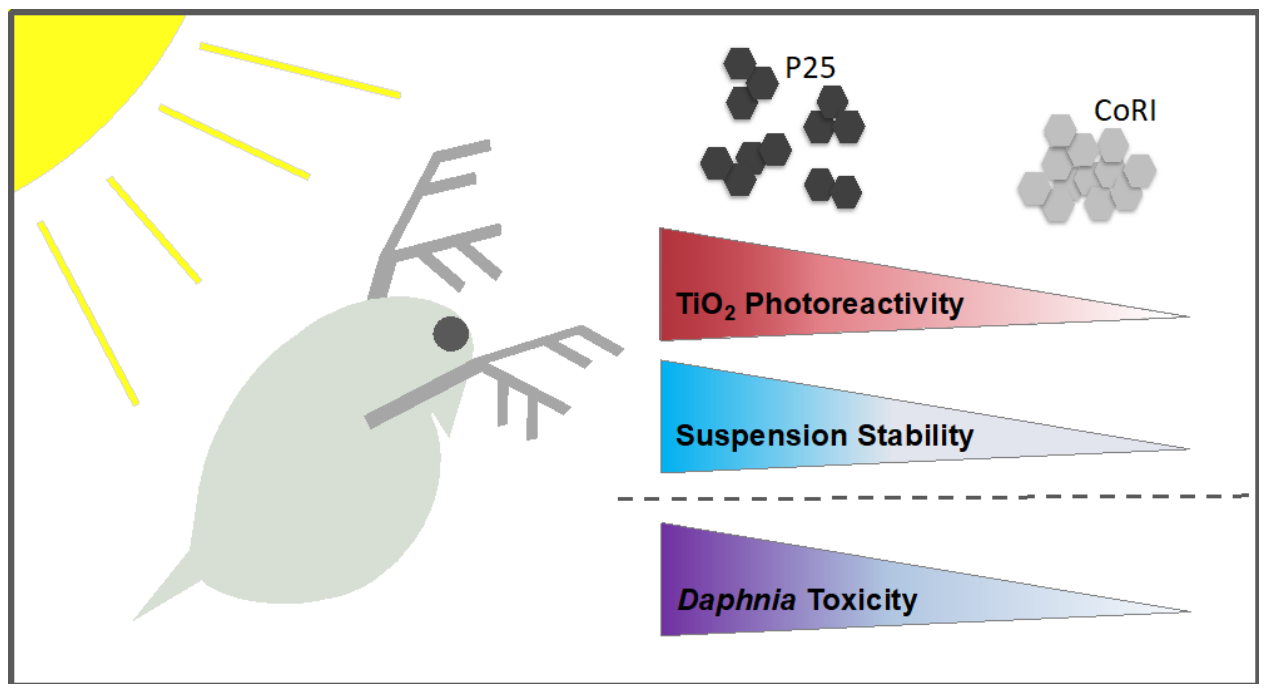


Daphnia magna toxicity is strongly influenced by the differences in photoreactivity and stability between two $\mathrm{TiO}_{2}$ nanoparticle formulations.

\section{Abstract}

Given their widespread manufacture and use, it is anticipated that titanium dioxide nanoparticles ( $\mathrm{TiO}_{2} \mathrm{NPs}$ ) will make their way into environmental surface waters where they may adversely impact biota. Characteristics of both the NPs themselves and the aquatic environment will determine the potential risks associated with release. Here, two different commercial formulations of $\mathrm{TiO}_{2}$ NPs are investigated in terms of aggregate stability, photoreactivity, and toxicity to the freshwater crustacean Daphnia magna. D. magna neonates were exposed to $\mathrm{TiO}_{2} \mathrm{NPs}$ in $48 \mathrm{~h}$ acute toxicity tests under either visible or UV illumination, in either the presence or absence of natural organic matter (NOM). Negligible lethal toxicity was observed by either $\mathrm{TiO}_{2}$ formulation when illuminated by visible light, although swimming performance was significantly affected. For both NPs, the presence of UV light dramatically increased hydroxyl radical $\left({ }^{*} \mathrm{OH}\right)$ generation and toxicity, although this was partially mitigated by the presence of NOM. Differences in toxicity between NPs are discussed in terms of stability and photoreactivity. These results show that not all $\mathrm{TiO}_{2}$ should be treated equally, and that differences in particle stability and photoreactivity must be taken into account when predicting risk.

\section{Introduction}

Titanium dioxide nanoparticles ( $\mathrm{TiO}_{2} \mathrm{NPs}$ ) comprise a significant fraction of global NP production and use at the industrial scale, with estimates on the order of thousands of tons per year ${ }^{1-3} \cdot \mathrm{TiO}_{2}$ NPs are widely used as additives in surface coatings, paints, and cosmetic products because of their optical properties 3,4 . Estimates suggest that 8-22 \% of NPs by 
mass end up in waters worldwide, during or after their use ${ }^{5}$. As a result, $\mathrm{TiO}_{2} \mathrm{NP}$ release and exposure of aquatic organisms is anticipated ${ }^{6,7}$. The presence of $\mathrm{TiO}_{2} \mathrm{NPs}$ originating from sunscreens has been observed in waters around public beaches at tens of $\mathrm{ppb}^{8}$, which is in keeping with estimates of expected environmental concentrations 5, 9, 10 .

Given the economic interest in and widespread use of $\mathrm{TiO}_{2}$, the ecotoxicity of $\mathrm{TiO}_{2} \mathrm{NPs}_{\text {sas }}$ been investigated with many animal models and algae ${ }^{11-13}$. D. magna is one common model that is particularly useful given its sensitivity and position at the base of the food web. To date, laboratory studies broadly indicate that for D. magna, the acute toxicity of nano-sized $\mathrm{TiO}_{2}$ is minimal for exposures at environmentally relevant concentrations under visible light. Reported toxic concentrations are often on the order of hundreds of ppm and vary widely ${ }^{14-}$ 18. Moreover, $\mathrm{TiO}_{2} \mathrm{NP}$ stability and toxicity have been shown to depend on aquatic chemistry $19,20$.

$\mathrm{TiO}_{2}$ is also an efficient photocatalyst, capable of producing reactive oxygen species (ROS) such as ${ }^{*} \mathrm{OH}$ when irradiated by UV light ${ }^{21}$. The band gap of anatase, the more photocatalytic form, is $3.2 \mathrm{eV}$, which corresponds to wavelengths less than $385 \mathrm{~nm}{ }^{22}$. For D. magna, studies that have considered the impact of UV light observe drastic increases in toxicity $23,24$. Mansfield et al. demonstrated that toxicity increases with the intensity of radiation using filtered natural sunlight ${ }^{25}$. Additionally, Wormington et al. highlighted the impact of natural organic matter (NOM) on ROS production and, thus, toxicity 26. Understanding how commercially relevant formulations of NPs will behave following release in terms of stability in the water column, photoreactivity, and ecotoxicity will improve our understanding of risk related to $\mathrm{TiO}_{2}$ release. While NP morphology and surface properties will impact reactivity 27, no direct comparisons between $\mathrm{TiO}_{2}$ types have been reported. Furthermore, toxicity 
studies often use different formulations of $\mathrm{TiO}_{2}$ and different exposure media to prepare suspensions, which complicates comparison.

In this study, we compare the stability and photoreactivity of two $\mathrm{TiO}_{2} \mathrm{NP}$ suspensions as they impact D. magna toxicity. D. magna exposed to $\mathrm{TiO}_{2} \mathrm{NPs}$ were illuminated by either visible or UV-A light, both in the presence and absence of NOM. Two formulations of $\mathrm{TiO}_{2}$ NPs were employed to assess their relative impacts on D. magna: the widely studied commercial P25 (80\% anatase, $20 \%$ rutile) (Evonik, Germany), and a proprietary formulation composed of pure anatase and used as a paint additive, as supplied by the Coatings Research Institute (CoRI, Belgium). Photoreactivity under the different conditions was quantified through the use of a specific hydroxyl radical probe and related to observed toxicity. This work highlights the importance of $\mathrm{TiO}_{2}$ formulation on photoreactivity, the stability of NP suspensions, and the toxicity of $\mathrm{TiO}_{2} \mathrm{NPs}$.

\section{Materials and Methods}

$\mathrm{TiO}_{2} \mathrm{NPs}:$

Experiments were performed with two formulations of $\mathrm{TiO}_{2}$ NPs. P25 Aeroxide (Evonik, Germany) is commonly studied in the literature and is a mixture of anatase and rutile at roughly an 80:20 ratio, respectively. The second $\mathrm{TiO}_{2} \mathrm{NP}$ (CoRI) is a proprietary formulation composed of $95+\%$ anatase and used as a paint additive (Coatings Research Institute, Belgium). Both formulations are comprised of uncoated $\mathrm{TiO}_{2} \mathrm{NPs} \mathrm{TiO}_{2} \mathrm{NP}$ stock suspensions were prepared at 50-200 ppm in EPA Moderately Hard Reconstituted Water (MHW) ${ }^{28}$, which consists of $96 \mathrm{mg} \mathrm{L}^{-1}$ sodium bicarbonate (99.7+\%, Sigma-Aldrich), $60 \mathrm{mg} \mathrm{L}^{-1}$ calcium sulfate dihydrate (98\%, Arcos Organics), $60 \mathrm{mg} \mathrm{L}^{-1}$ magnesium sulfate (Fisher Scientific), and $4 \mathrm{mg} \mathrm{L}^{-1}$ potassium chloride (USP, Fisher Scientific). The resulting water is designed to mimic 

was identified as the $\mathrm{pH}$ for which the EPM of a suspension was zero. s off) for 6 min total following the NIST protocol by Taurozzi et al. ${ }^{29}$.

\section{Characterization:}

natural, moderately hard water with a $\mathrm{pH}$ of 7.8. Immediately prior to use, stock suspensions were probe sonicated (Q700, QSonica, Newton, Connecticut, USA) in pulse mode (12 s on, 3

X-ray photoelectron spectroscopy (Thermo Scientific) was used for elemental identification of $\mathrm{TiO}_{2} \mathrm{NPs}$. A monochromated $\mathrm{Al}-\mathrm{K} \alpha \mathrm{X}$-ray source was used with a pass energy of $200 \mathrm{eV}$ and a step width of $1 \mathrm{eV}$ for the survey spectra and a pass energy of $50 \mathrm{eV}$ and a step width of $0.1 \mathrm{eV}$ for the detailed spectra. Scans were performed with a spot size of $200 \mu \mathrm{m}$ and the flood gun on in a $10^{-8}$ mbar vacuum. The aggregate size of $\mathrm{TiO}_{2}$ suspensions was determined by Dynamic Light Scattering (DLS) with a Zetasizer Nano ZS (Malvern, Massachusetts, USA). Size measurements are reported both as intensity weighted hydrodynamic diameter and Zaverage diameter (cumulants mean diameter). The heterogeneity in aggregate sizes within a suspension is indicated by the polydispersity index (PDI), which ranges from 0 to 1 , with more polydisperse samples approaching unity. DLS measurements were confirmed using Transmission Electronic Microscopy (Philips CM200 TEM, Advanced Microscopy Techniques Corp.) performed on suspensions prepared on lacey carbon grids (CF400-CU, Electron Microscopy Sciences) and imaged at $200 \mathrm{kV}$. Electrophoretic mobility (EPM) measurements were collected via laser doppler velocimetry and converted to zeta potential (ZP) using the Henry equation (ZetaSizer Nano ZS, Malvern). To determine the isoelectric point (IEP), pH titrations were performed in duplicate using $100 \mathrm{~mL}$ suspensions of $10 \mathrm{ppm}$ P25 $\mathrm{TiO}_{2}$ in MHW. Suspensions were continually stirred while pH was monitored and adjusted with $0.1 \mathrm{M} \mathrm{HCl}$. EPM was measured at each pH step, and the isoelectric point (IEP) 
$124 \mathrm{TiO}_{2}$ concentrations in suspension over time were measured via ICP-MS following 125 microwave digestion (MARS 6, CEM Corp, North Carolina, USA) in sulfuric acid. $100 \mathrm{~mL}$ 126 samples were prepared using the same protocol as acute toxicity tests (below) and 127 undisturbed over the $48 \mathrm{~h}$ measurement period. $2 \mathrm{~mL}$ aliquots were taken periodically from 128 mid height in the water column, to which $3 \mathrm{~mL}$ high purity sulfuric acid (96+\%, Arcos 129 Organics) was added. Samples were microwave digested at $1400 \mathrm{~W}$ following a $30 \mathrm{~min}$ ramp 130 to $200{ }^{\circ} \mathrm{C}$ and hold time of $40 \mathrm{~min}$. Following digestion, samples were diluted to a final acid 131 concentration of $4 \% \mathrm{v} / \mathrm{v}$ and analyzed via ICP-MS (NexION 300X, Perkin Elmer, 132 Massachusetts, USA). Calibrations were performed with Ti-47 isotope using the same acid 133 matrix as the samples. Sc-45 was used as an internal standard and variation was $\pm 10 \%$. 134 Detection limits were $0.02 \mu \mathrm{g} \mathrm{L}-1$ and background equivalent concentrations were $0.095 \mu \mathrm{g}$ $135 \mathrm{~L}^{-1}$. Method spikes in MHW undergoing the full digestion method using a Ti-47 NIST standard 136 provided recoveries of $76 \%$.

$\mathrm{TiO}_{2}$ Photoreactivity Measurements:

139 Photoreactivity measurements were performed under either visible (8 W fluorescent 140 6500K-daylight bulbs, Eiko Global, Kansas, USA) or UV light (8 W fluorescent UV lamps, peak 141 output $365 \pm 15 \mathrm{~nm}$, Hikari Lamps, California, USA). The output and modeled spectra of the 142 UV bulbs is shown in Figure S1. Samples were located under 6 bulbs placed in parallel. 143 Because the intensity of light was greater towards the center of the bulbs, the distance from 144 the bulbs to each beaker for UV exposure was adjusted between 15.2 and $20.3 \mathrm{~cm}$ to achieve 145 the desired irradiance of $13.0 \pm 0.1 \mathrm{~W} \mathrm{~m}^{-2}$, as measured with a UV-radiometer (MU-200, 146 Apogee Instruments). This UV intensity was selected as it resulted in an irradiance lower 147 than full sun exposures, as may be encountered during cloudy days or in partially shaded 148 waters, and it minimized the likelihood of observed D. magna toxicity arising solely from the 
UV exposure. For comparison, a UV exposure due to sunlight of $32.4 \mathrm{~W} \mathrm{~m}^{-2}$ over the same wavelengths $(340-390 \mathrm{~nm})$ has been reported at $38^{\circ} \mathrm{N}$ on a clear summer day; the total UV irradiance was $51.9 \mathrm{~W} \mathrm{~m}^{-2}(290-400 \mathrm{~nm})^{30}$. Visible light exposures were performed under 4 lamps at a height of $35.6 \mathrm{~cm}$ and intensity of $12 \mu \mathrm{mol} \mathrm{m}^{-2} \mathrm{~s}^{-1}$, measured with a MQ-200 light meter (Apogee Instruments). For visible light exposures, a small amount of UV radiation was observed (0.1-0.2 $\mathrm{W} \mathrm{m}^{-2}$, two orders of magnitude less than the irradiance used in the UV exposures).

The production of hydroxyl radicals $\left({ }^{*} \mathrm{OH}\right)$ was monitored over $48 \mathrm{~h}$ using $0.5 \mathrm{mM}$ terephthalic acid (TA, 99+\%, Arcos Organics). Hydroxylation of the molecule produces the fluorescent 2-hydroxy terephthalic (2-HTA, 97\%, Sigma-Aldrich) (ex $315 \mathrm{~nm} / \mathrm{em} 425 \mathrm{~nm}$ ). ${ }^{*} \mathrm{OH}$ generation under either visible or UV light was monitored for $10 \mathrm{ppm} \mathrm{TiO}_{2}$ in $\mathrm{MHW}$ for both P25 and CoRI. For photoreactivity tests, $10 \mathrm{ppm}$ sonicated $\mathrm{TiO}_{2}$ in either the presence or absence of $10 \mathrm{ppm}$ NOM was placed in MHW in $250 \mathrm{~mL}$ polypropylene beakers (Fisher Scientific). Suspensions were kept undisturbed in the dark at room temperature and allowed to aggregate and settle naturally. At $0,6,12,24$, and $48 \mathrm{~h}$, suspensions were exposed to UV light in triplicate for $1 \mathrm{~h}$ with $1.2 \mathrm{~mL}$ aliquots taken every $10 \mathrm{~min}$ at mid height in the beaker. Suspensions were sacrificial (i.e., each beaker was only exposed to UV light once). Visible light exposures were only performed immediately after sonication $(\mathrm{t}=0 \mathrm{~h})$. Sampled aliquots were centrifuged for $5 \mathrm{~min}$ at 12,000 rpm (9660g) (MiniSpin, Eppendorf), and the supernatant was analyzed for fluorescence intensity (Fluoromax-4, Horiba Jobin Yvon). For samples containing NOM, Suwannee River NOM (Reverse Osmosis Isolate, International Humic Substances Society, USA) was added to the NP suspensions at $10 \mathrm{ppm}$. This concentration was selected as it is mid-range in concentrations common to surface waters 31. Controls consisted of MHW and MHW with 10 ppm NOM. Fluorescence was converted to 
${ }^{*} \mathrm{OH}$ concentration using a standard curve of 2 -HTA from $0.00625-0.125 \mu \mathrm{M}$, assuming a trapping efficiency of $80 \%{ }^{32}$.

The absorbance of NOM in solution at concentrations ranging from 1.25 to 100 ppm was measured via UV-Visible spectroscopy (Agilent 8453) using a $1 \mathrm{~cm}$ path length quartz cuvette with DI water as the blank to determine the impact of NOM on UV light penetration in the water column. The measured absorbance was then used to determine the absorption coefficient, according to the Beer-Lambert Law and the intensity of light as a function of depth was modeled.

\section{D. magna Growth:}

D. magna were obtained from Environment and Climate Change Canada and grown in MHW. $2 \mu \mathrm{g} \mathrm{L}^{-1}$ sodium selenate (Fisher Scientific, >99\%) and $2 \mu \mathrm{g} \mathrm{L}^{-1}$ vitamin B12 (Fisher Scientific, 98\%) were added to rear water as advised by Environment and Climate Change Canada to maintain the health of the colony ${ }^{33}$. These supplements were not included in test water to prevent interference with generated reactive species. D. magna were reared at $21 \pm 1^{\circ} \mathrm{C}$ and exposed to a $16 \mathrm{~h}$ light, $8 \mathrm{~h}$ dark cycle following OECD guidelines ${ }^{34}$. D. magna were fed daily with a mixture of Chlamydomonas reinhardtii $\left(3.0 \times 10^{6}\right.$ cells $\left./ \mathrm{mL}\right)$ and Yeast Cerophyll ${ }^{\mathrm{MC}}$ Trout (YCT) $(0.8 \%$ v/v) (Aquatic Research Organisms Inc., New Hampshire, USA).

\section{Acute Toxicity Testing:}

Acute $(48 \mathrm{~h})$ toxicity tests were conducted according to OECD guidelines ${ }^{35}$. Each replicate consisted of 5 neonates $(<24 \mathrm{~h}$ in age) placed in MHW containing various concentrations of freshly sonicated $\mathrm{TiO}_{2}$ NPs and NOM (100 mL total volume) and exposed to either visible or UV-A light. Illumination during testing followed the same $16 \mathrm{~h}$ light, $8 \mathrm{~h}$ dark cycle used 
during rearing. The concentrations tested were randomized over time, and control samples containing 5 neonates in MHW only or MHW plus 10 ppm NOM were run with each test. The 201 addition of $10 \mathrm{ppm}$ NOM was not observed to cause mortality. Temperature, $\mathrm{pH}$, and 202 dissolved oxygen content were measured in control and representative samples at the 203 beginning and end of each test. All values conformed to OECD guidelines. Test results for a 204 given replicate were discarded if mortality was observed in the associated control samples. 205 At 24 and 48 h, D. magna were assessed and characterized as healthy, immobilized, or dead. 206 Visual inspection of D. magna was performed at 10× optical microscopy (Stereomaster, 207 Fisher Scientific). At least 4 replicates of each condition were conducted. Calculations of LC50 were performed on tests by fitting a sigmoidal function to the data using the Hill equation.

Swimming Performance:

211 Differences in D. magna swimming performance after exposure to visible light and $\mathrm{TiO}_{2}$ NPs 212 were assessed by measuring the distance traveled in one minute following the procedure 213 outlined by Pikuda et al. ${ }^{36} .10$ neonates were placed in polypropylene beakers holding 100 $214 \mathrm{~mL}$ suspensions containing $1 \mathrm{ppm} \mathrm{TiO}_{2}$ and exposed to the same conditions as the acute toxicity tests. After 48 h, 5 visibly healthy daphnids (i.e. freely swimming) were individually selected, placed one at a time in a small volume (1 mL) of MHW in a glass bottom culture dish 217 containing a $1.4 \mathrm{~cm}$ diameter microwell (MatTek Corp), and allowed to acclimate for $2 \mathrm{~min}$ before being recorded for $1 \mathrm{~min}$. The distance traveled by each daphnid was calculated using the Kinovea motion tracking software (www.kinovea.org). Each test was run in triplicate, 220 resulting in 15 swimming paths collected per condition. MHW and MHW + NOM controls 221 were run with each condition ( $\mathrm{n}=30$ for each). Statistical significance between treatments was determined by 1-way ANOVA followed by post-hoc Tukey's HSD $(\mathrm{p}<0.05)$. 


\section{Results and Discussion}

\section{$\mathrm{TiO}_{2}$ Characterization}

K-Alpha XPS was performed on P25 and CoRI NPs deposited onto carbon tape. Figure S2 shows XPS survey scans, which did not identify the presence of any elements other than carbon (attributed to the carbon tape), oxygen and titanium for either NP, suggesting both NPs are pure $\mathrm{TiO}_{2}$. NPs were imaged by TEM (Figure 1), and results are listed in Table 1. ImageJ analysis of TEM images indicates that the size of the primary particles differs greatly between P25 $(29 \pm 8 \mathrm{~nm})$ and CoRI $(5 \pm 1 \mathrm{~nm})(\mathrm{n}=100)$. Figures $1 \mathrm{c}$ and g illustrate the crystallinity of the both types of Ti02. As can be see, the lattice structure of P25 is more coherent over longer distances owing to the larger primary particle size. For P25, lattice spacings of both $0.33 \mathrm{~nm}$ (corresponding to the 110 Miller index of rutile) and $0.35 \mathrm{~nm}$ spacings (corresponding to the 101 Miller index of anatase) are observed. For CoRI, only 0.35 nm spacings (anatase) are observed.

Selected area electron diffraction (SAED) patterns for P25 and CoRI are shown in Figure 1d and $h$, respectively. The ring patterns are characteristic of polycrystalline samples. Analysis of the d-spacing was performed using an external calibration with aluminum diffraction standard. Identified d-spacings from diffraction rings along with their corresponding Miller indices are given in Table S3. Both $\mathrm{TiO}_{2}$ samples exhibit diffraction patterns corresponding to anatase. The diffraction rings for CoRI are less distinct than those for P25, which could be indicative of the smaller size of CoRI NPs and electron beam passing through more areas of the NP that are amorphous (i.e. at the outer edges of the NP where lattice breaks down). That the diffraction rings of P25 also correspond to anatase is likely a function of the relative amounts of anatase $(80 \%)$ and rutile $(20 \%)$ in the formulation. Thus, the majority of 
diffraction will be due to anatase, and diffraction corresponding to rutile may lie in the few

points that can be observed between the more intense anatase rings.

DLS results shown in Table 1 highlight further differences between the suspensions. CoRI

252 NPs form very polydisperse suspensions with a large range of sizes, as evidenced by both the

253 high polydispersity index ( $>0.80$ for both CoRI and CoRI + NOM) and the difference between

254 the Z-average diameter (order of $\mu \mathrm{m}$ ) and the intensity weighted average diameter

255 (hundreds of $\mathrm{nm}$ ) (see Supporting Information for further discussion) ${ }^{37}$. While DLS is not

256 recommended for accurate sizing of highly polydisperse suspensions ${ }^{38}$, and results for CoRI

257 NPs should be viewed accordingly, comparing results between the two NPs can provide

258 insight into the differences between the two nanomaterials. The PDI of P25 and P25 + NOM

259 is less than 0.25 , suggesting these suspensions are much more monodisperse than CoRI and

260 CoRI + NOM. Also, both the Z-average diameter and intensity weighted average diameter are

261 between 140-240 nm, in agreement with previous observations ${ }^{29,39}$. Thus, for the same

262 aquatic chemistry and the same dispersion method, suspensions of CoRI NPs are comprised

263 of a large range of aggregate sizes whereas aggregates of P25 NPs are generally smaller and

264 much more monodisperse.

265

266

267

268

269

\begin{tabular}{|c|c|c|c|c|c|c|c|}
\hline & {$\left[\mathrm{TiO}_{2}\right]$} & {$[\mathbf{N O M}]$} & TEM & \multicolumn{2}{|c|}{ DLS } & ZP \\
\cline { 2 - 8 }$(\mathrm{mg} / \mathrm{L})$ & $(\mathrm{mg} / \mathrm{L})$ & $\begin{array}{c}\text { Primary } \\
\text { particle size } \\
\text { (d.nm) }\end{array}$ & $\begin{array}{c}\text { Z-average } \\
\text { size } \\
\text { (d.nm) }\end{array}$ & $\begin{array}{c}\text { Intensity } \\
\text { weighted } \\
\text { size (d.nm) }\end{array}$ & PDI & $(\mathrm{mV})$ \\
\hline P25 & 5 & 0 & $29 \pm 8$ & 167 & 188 & 0.21 & $-16.4 \pm 0.7$ \\
\hline P25 + NOM & 5 & 10 & & 142 & 233 & 0.22 & $-18.8 \pm 0.8$ \\
\hline CoRI & 5 & 0 & $5 \pm 1$ & 2598 & 354 & 0.86 & $-20.1 \pm 0.7$ \\
\hline CoRI + NOM & 5 & 10 & & 2467 & 379 & 0.82 & $-22.0 \pm 1.4$ \\
\hline
\end{tabular}

Table 1. $\mathrm{TiO}_{2}$ NP characterization. All suspensions were prepared in EPA MHW and measurements performed directly after sonication $(\mathrm{t}=0 \mathrm{~h})$. Reported DLS values are averages of triplicate measurements. See Table S3 for individual results. 

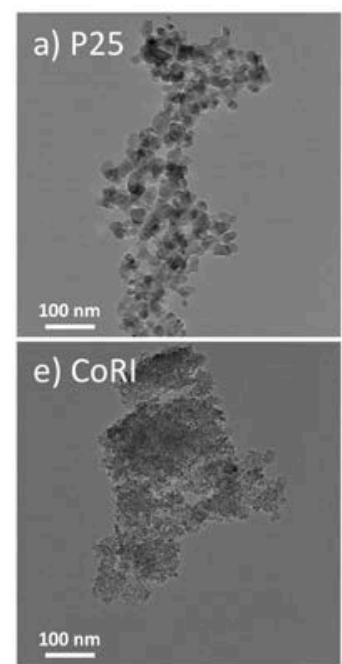
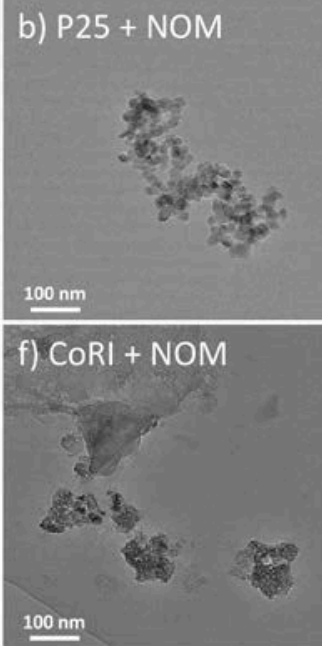
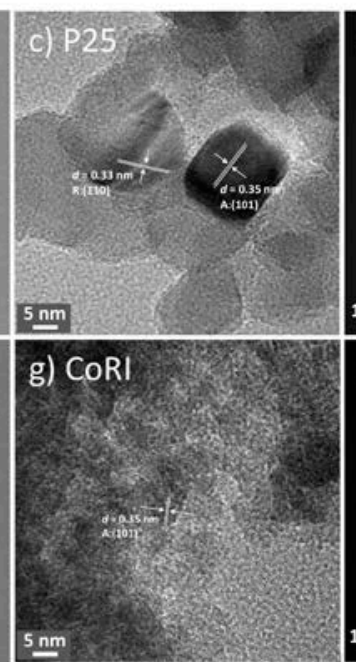

d)

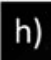

h)

$1001 / \mathrm{nm}$
Figure 1. TEM images of $\mathrm{TiO}_{2}$ NPs suspended at 5 ppm in MHW: a) bare P25, b) P25 in 10 ppm NOM, c) high resolution of P25, d) ring diffraction from P25 e) bare CoRI, f) CoRI in 10 ppm NOM, g) high resolution of CoRI, h) ring diffraction of CoRI.

In all test conditions, measurements of EPM indicated negatively charged particles, which was expected given the $\mathrm{pH}$ of $\mathrm{MHW}$ at 7.8 is above the point of zero charge (pzc) of $\mathrm{TiO}_{2}$ (approx. 6 for bare anatase) ${ }^{40}$. Considerable differences were observed in the isoelectric points (IEPs) of P25 and CoRI in MHW in this study. ZP vs pH titrations are shown in Figure S5. The IEP of P25 was observed to be approximately 5.8, which agrees well with previous results for P25 in the presence of sulfate (where the IEP of P25 in nitrate or chloride was 6.6) 39. In contrast, the IEP of CoRI was not observed, occurring at a $\mathrm{pH}<3$. While the difference in the ZP vs pH titrations could be indicative of differences in surface coatings or surface passivation, XPS results do not show the presence of any other elements beyond titanium and oxygen. These deviations may be due to the anionic species present in MHW, namely carbonate and sulfate. Previously, both anions were observed to undergo inner sphere ligand exchange and alter the IEP of P25, with carbonate having a particularly strong impact ${ }^{39}$. The shift in IEP suggests that CoRI NPs may more readily interact with carbonate than P25. The presence of NOM had a negligible impact on surface charge (Table 1). 


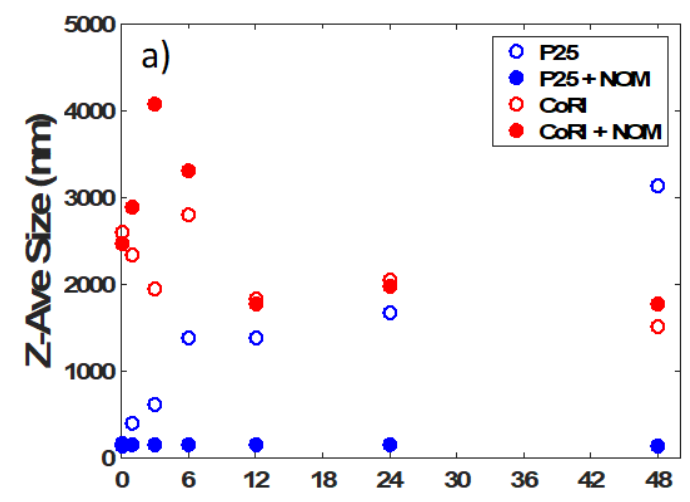

Time (h)

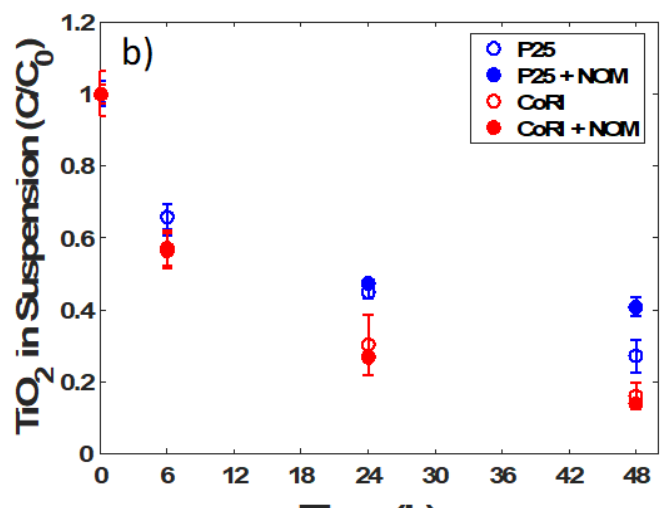

Time (h)

Figure 2. a) Time-resolved DLS data of $\mathrm{TiO}_{2}$ suspensions. Aggregate size versus time for P25 or CoRI NPs suspended in MHW or MHW supplemented with 10 ppm NOM. b) Normalized ICP-MS measurements of $\mathrm{TiO}_{2}$ concentration remaining in suspension (measured as Ti) versus time.

Time resolved DLS (TRDLS) data, plotted as Z-average size (Figure 2a), indicate that the $\mathrm{TiO}_{2}$ suspensions exhibit a range of stabilities. P25 NPs, though initially forming small aggregates, are largely unstable in MHW and undergo significant aggregation over the first $6 \mathrm{~h}$. The zaverage size increases from approximately $160 \mathrm{~nm}$ initially to the micron range after $48 \mathrm{~h}$. However, in the presence of 10 ppm NOM (P25 + NOM), the P25 NPs are quite stable, with no change in DLS size over the $48 \mathrm{~h}$ period. Despite this, a decrease in count rate (Figure S6) suggests that some deposition occurs as aggregates begin to settle outside the path of the laser, resulting in the decreased scattering of incident photons. Z-average values for CoRI NP aggregates are initially much larger, on the order of microns, but decrease over time. Given that samples are not stirred, and thus breakup of aggregates is not expected, these results suggest significant settling of the largest fraction over time (Figure 2a). In contrast to P25, the presence of NOM had no discernable impact on CoRI stability.

To further determine the stability of the $\mathrm{TiO}_{2}$ NPs in MHW, ICP-MS was performed on aliquots sampled at $0,6,24$, and $48 \mathrm{~h}$ from mid height in a $250 \mathrm{~mL}$ polypropylene beaker (Figure 2b). The concentration of all NPs, nominally $1 \mathrm{ppm}$ at $\mathrm{t}=0 \mathrm{~h}$, drastically decreased 
313

over time. In agreement with TRDLS results, P25 + NOM exhibited the least amount of loss,

314 with $41 \pm 3 \%$ remaining in the water column, compared to P25 by itself ( $27 \pm 5 \%$ ). CoRI NPs were less stable than P25 NPs. After 48 h, $16 \pm 4 \%$ and $14 \pm 2 \%$ of CoRI and CoRI + NOM remained in the water column, respectively. These results agree with observations by Ma et al. who report up to $67 \%$ loss over $24 \mathrm{~h}$ with P25 in MHW at a starting concentration of 7 $\operatorname{ppm}^{24}$. was visually observed as a white deposit on the bottom of beakers.

\section{$\mathrm{TiO}_{2}$ Photoreactivity}

NP photoreactivity tests were performed using the same lighting conditions employed

334 during toxicity testing. ${ }^{*} \mathrm{OH}$ were detected by measuring the increase in fluorescence 335 resulting from the hydroxylation of TA to 2-HTA. These fluorescence measurements were 336 then converted to ${ }^{*} \mathrm{OH}$ generation rates through the use of a 2-HTA standard curve (Figure

Thus, for all conditions, the concentration of $\mathrm{TiO}_{2} \mathrm{NPs}$ is expected to decrease over time during 48 h D. magna exposures. The influence of NOM may be to stabilize the smallest fraction, limiting aggregation, though particles will still be subject to settling. For P25, where the initial suspension is made of smaller, more homogeneous aggregates, this influence is more pronounced. Calculations of Stokes' settling velocities (Figure S7) suggest that aggregates larger than $950 \mathrm{~nm}$ (diameter) will settle the full distance of the water column $(13.8 \mathrm{~cm})$ over $48 \mathrm{~h}$. Aggregates larger than $675 \mathrm{~nm}$ will settle half the height of the water column in $48 \mathrm{~h}$. While some resuspension and bioturbation would be expected due to the swimming action of D. magna in the beaker, the $48 \mathrm{~h}$ duration of the test suggests that most particles will be susceptible to settling. At the highest $\mathrm{TiO}_{2} \mathrm{NP}$ concentrations, settling of $\mathrm{TiO}_{2}$ 

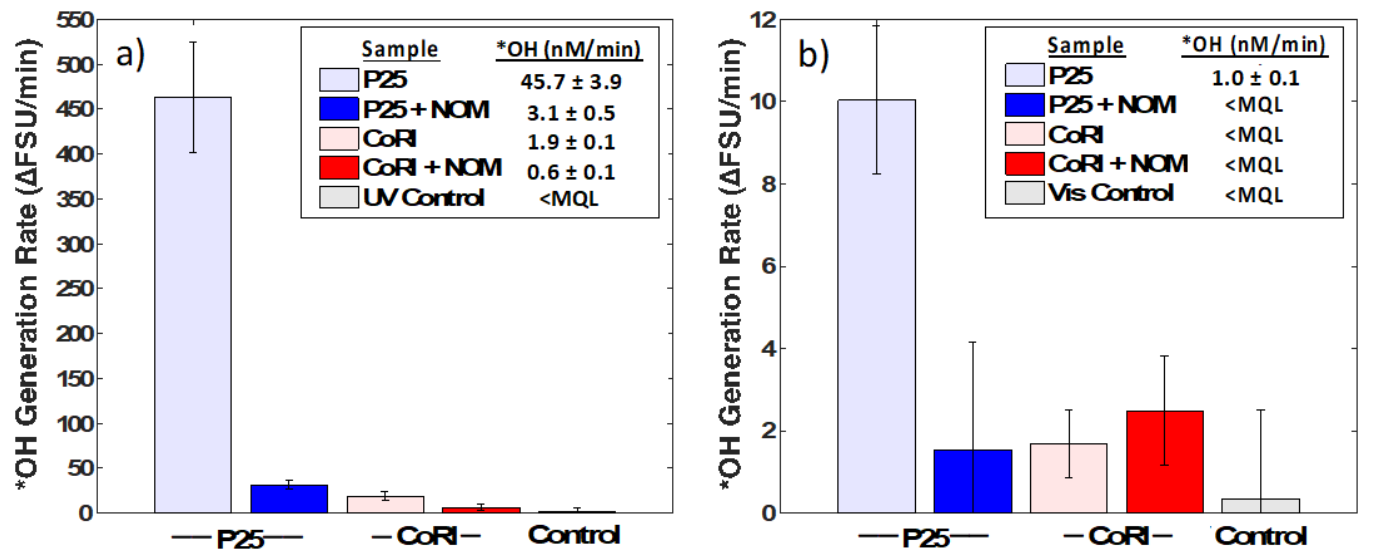

Figure 3. ${ }^{*} \mathrm{OH}$ generation rates plotted as change in fluorescence over time for $10 \mathrm{ppm}^{\mathrm{TiO}} 2$ as a function of NP type and presence/absence of NOM under a) UV light and b) visible light conditions used in D. magna toxicity tests. ${ }^{*} \mathrm{OH}$ generation rates $(\mathrm{nM} / \mathrm{min})$ calculated from 2-HTA standard curves are listed in the legends. Samples for which fluorescence values were below the minimum quantification limit are listed as $<$ MQL. Note, y-axis scales are different, with P25 generating greater * $\mathrm{OH}$ than CORI.

The presence of NOM reduced initial ${ }^{*} \mathrm{OH}$ production by nearly an order of magnitude for both types of NPs to $3.1 \pm 0.5$ and $0.6 \pm 0.1 \mathrm{nM} \mathrm{min}^{-1}$ for P25 + NOM and CoRI + NOM, respectively. As TA provides a measure of ${ }^{*} \mathrm{OH}$ in the bulk solution ${ }^{41}$, a reduction in et al. showed that, in a $250 \mathrm{~mL}$ crystallizing dish, $10 \mathrm{ppm}$ NOM was observed to reduce UV intensity by $17 \%$, providing evidence that the primary impact of NOM is due to ROS quenching 26 .

By measuring the absorbance of NOM at multiple concentrations in solution, we calculated 
intensity as a function of depth in the beaker (see Supporting Information, Figure S10). In

361 the presence of NOM, the total intensity of UV light at the bottom of the beaker $\left(3.71 \mathrm{~W} \mathrm{~m}^{-2}\right)$

362 is only $28.5 \%$ that of the upper surface. The spatial average of UV light intensity in the water

363 column was calculated to be $7.58 \mathrm{~W} \mathrm{~m}^{-2}$, or $57.9 \%$ the intensity at the surface. The

364 contribution to light attenuation from MHW itself was negligible. Using these calculations

365 and the observed ${ }^{*} \mathrm{OH}$ generation rate of $45.7 \mathrm{nM} \mathrm{min}^{-1}$ for $\mathrm{P} 25$, some insight into the relative

366 impact of light attenuation and ${ }^{*} \mathrm{OH}$ quenching can be obtained. If a decrease in ${ }^{*} \mathrm{OH}$

367 production was due solely to light attenuation, the generation rate of P25 + NOM would be

368 anticipated to be $26.5 \mathrm{nM} \mathrm{min}^{-1}$. The measured rate of $3.1 \pm 0.5 \mathrm{nM} \mathrm{min}^{-1}$ is substantially less

369 than what the decrease in UV intensity would merit, suggesting a significant contribution

370 due to radical quenching. Both light attenuation and quenching will produce the same effect:

371 a decreased delivery of the radical to a given target (e.g., D. magna). While NOM is itself a

372 known photocatalyst, capable of producing ROS (e.g., singlet oxygen ${ }^{42}$ ), no ${ }^{*} \mathrm{OH}$ production

373 was detected in controls containing only $10 \mathrm{ppm}$ NOM. Overall, * $\mathrm{OH}$ generation under UV

374 light followed the trend of P25 > P25 + NOM > CORI > CoRI + NOM.

${ }^{*} \mathrm{OH}$ production was measured over $48 \mathrm{~h}$ in beakers that were otherwise undisturbed, 377 allowing $\mathrm{TiO}_{2}$ NPs to aggregate and settle naturally (Figure 4). The results show the 378 anticipated evolution in * $\mathrm{OH}$ exposure for D. magna over time. For CoRI and P25 NPs in the 379 absence of NOM, a strong decrease in ${ }^{*} \mathrm{OH}$ generation over time is observed that agrees with 380 measured concentration and aggregation data. While the generation rate is less for NPs + 381 NOM, the decrease over time is not as pronounced. In particular, P25 + NOM, which after 48 $382 \mathrm{~h}$ had the largest remaining concentration in suspension $(41 \pm 3 \%)$ showed no change in * $\mathrm{OH}$ 383 production over time. Because ROS production is inversely correlated to the size of aggregate, 384 the smallest aggregates will be responsible for the majority of ROS production, and the net 
ROS production of a suspension will decrease as NPs aggregate 43,44 . Thus, the average concentration of ${ }^{*} \mathrm{OH}$ in suspension will decrease over time due to both the aggregation and settling of NPs. It's likely that NOM is stabilizing the smallest fraction of aggregates which then continue to produce ${ }^{*} \mathrm{OH}$ at roughly the same rate over $48 \mathrm{~h}$. Despite this stabilization, even at $48 \mathrm{~h},{ }^{*} \mathrm{OH}$ production for both P25 and CoRI in the absence of NOM remains greater than in the presence of NOM.

*OH production was also monitored under visible light exposure, with only P25 resulting in $\mathrm{a} * \mathrm{OH}$ generation rate $\left(1.0 \pm 0.1 \mathrm{nM} \mathrm{min}^{-1}\right)$ that was statistically different from the control ( $\mathrm{p}$ $<0.05)$. All other conditions were below the minimum quantification limit $(<M Q L)$ of the standard curve (Figure 3b). This small amount of radical generation in P25 likely arises due to the slight emission of the fluorescent bulbs that exists at energies above the band gap of
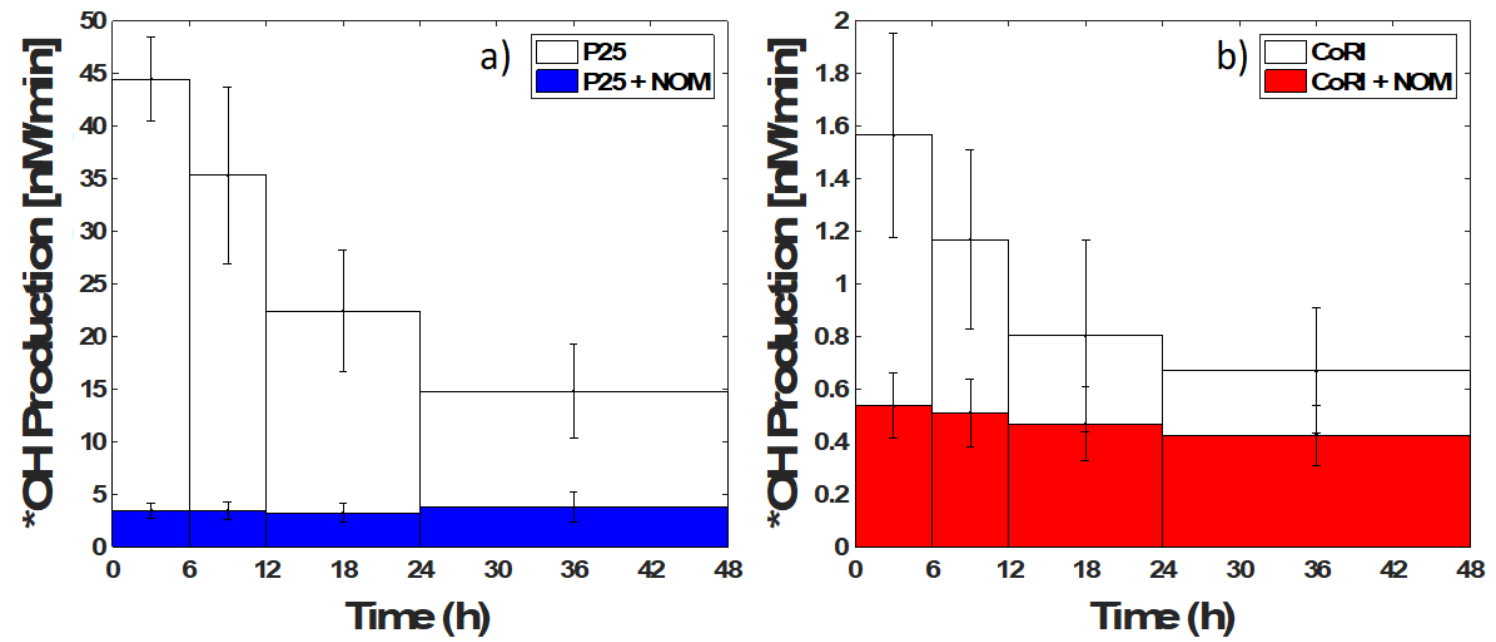

Figure 4. ${ }^{*} \mathrm{OH}$ generation rates $(\mu \mathrm{M} / \mathrm{min})$ for a) $10 \mathrm{ppm}$ P25 and b) CoRI over $48 \mathrm{~h}$ as a function of NP type and presence/absence of NOM under UV light $\left(13.1 \mathrm{~W} / \mathrm{m}^{2}\right)$. Values shown are averages of the measured generation rates \pm std. Note, $y$-axis scales are different, with P25 generating greater * $0 H$ than CORI.

405 
D. magna exposed to $\mathrm{TiO}_{2} \mathrm{NPs}$ under visible light displayed little toxicity (Figure 5a). Rapid aggregation and sedimentation were observed for bare $\mathrm{TiO}_{2}$ suspensions, with visible

409 deposits forming at the bottom of the beakers after a few hours for concentrations of $50 \mathrm{ppm}$ 410 and higher. When observing D. magna, care was taken not to disturb or mix the beakers so

411 as to limit resuspension of aggregated and settled NPs. For P25, a low level of toxicity was 412 observed at concentrations as low as $1 \mathrm{ppm}$, though this did not greatly increase even at 100 413 ppm. The maximum mortality was observed to be roughly $50 \%$ after $48 \mathrm{~h}$ in 100 ppm P25. 414 The addition of NOM to suspensions reduced the observed mortality, with only minimal $(<$ $41510 \%$ ) effects observed for P25 + NOM at 10 and 25 ppm TiO2. For CoRI NPs, slight toxicity 416 (up to 20\%) was observed at concentrations above $10 \mathrm{ppm}$; however, again this effect was 417 mitigated by the presence of NOM. No toxicity was observed at any concentration for CoRI + 418 NOM.

420 In the absence of UV light, any observed toxicity should be due to the particles themselves 421 rather than their photocatalytic ability. Although these results would appear to suggest that 422 P25 may be slightly more toxic than CoRI, the impact of P25 may be due to the observed low 423 levels of ${ }^{*} \mathrm{OH}$ generated under visible light. The fact that toxicity was observed for CoRI NPs 424 only above $10 \mathrm{ppm}$ supports that there is little inherent acute toxicity due to the $\mathrm{TiO}_{2} \mathrm{NPs}$ 425 themselves and agrees with previous reports where acute toxicity is only observed at unrealistically elevated concentrations $14,16,18$. 

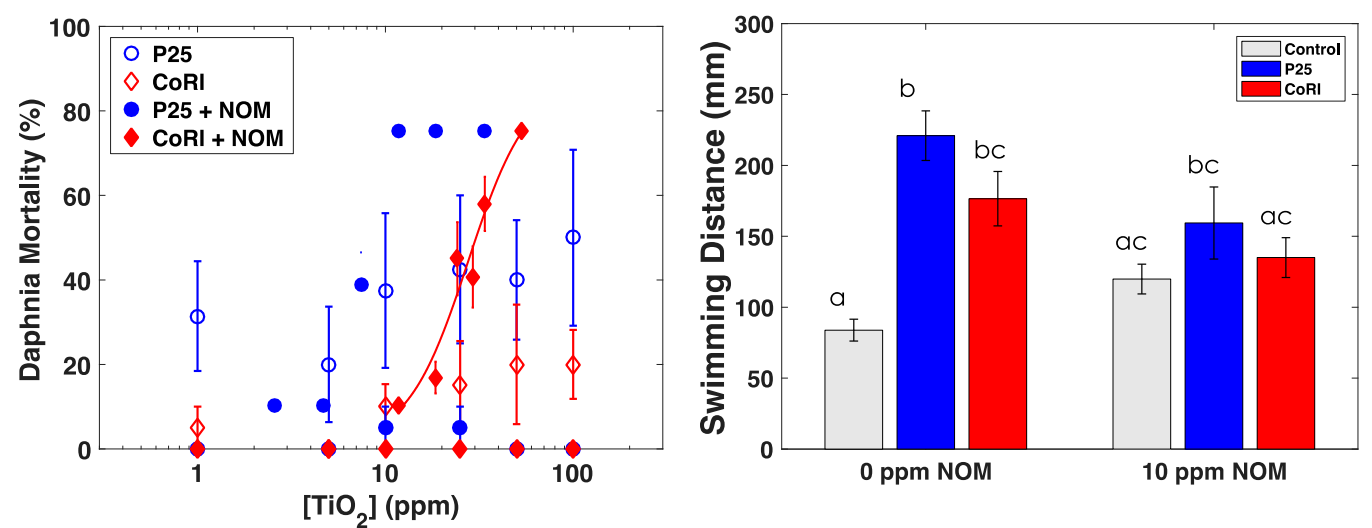

Figure 5. a) D. magna mortality after $48 \mathrm{~h}$ exposure to NPs under visible light. Error bars represent standard error ( $\mathrm{n} \geq 4)$, b) Distance covered by D. magna in $1 \mathrm{~min}$ following $48 \mathrm{~h}$ exposure to $1 \mathrm{ppm} \mathrm{TiO}_{2}$ and visible light. Error bars are standard error ( $\mathrm{n}=15$ for P25 and CoRI, $\mathrm{n}=30$ for controls). Letters denote statistical differences $(\mathrm{p}<0.05)$ determined by 1-way ANOVA followed by post-hoc Tukey's HSD.

\section{Swimming Performance}

While $100 \%$ mortality was not observed for $\mathrm{TiO}_{2}$ under visible light at any of the concentrations studied, other sublethal toxicity metrics such as swimming performance provide more sensitive endpoints for analyzing impact ${ }^{45}$. Figure $5 \mathrm{~b}$ presents the average distance covered in 1 min by D. magna following $48 \mathrm{~h}$ exposure to $1 \mathrm{ppm} \mathrm{TiO}_{2} \mathrm{NPs}$ under visible light, and example swimming traces are shown in Figure S12. D. magna exposed to only $\mathrm{TiO}_{2} \mathrm{NPs}$ exhibited statistically greater swimming activity $(221.0 \pm 17.5 \mathrm{~mm}$ and 176.5 \pm 19.2 for P25 and CoRI, respectively) than D. magna in only MHW (83.8 $\pm 7.7 \mathrm{~mm}$ ) (values reported as average \pm standard error). The difference was not significant, however, between $\mathrm{TiO}_{2}$ NPs + NOM $(159.4 \pm 25.4 \mathrm{~mm}$ and $135.0 \pm 14.0$ for P25 and CoRI, respectively $)$ and the MHW + NOM control $(127.6 \pm 16.1 \mathrm{~mm})$. Thus, the presence of $\mathrm{TiO}_{2}$ alone causes an increase in swimming activity, which may be a result of the D. magna attempting to escape a low level stressor, resulting in an increased depletion of energy reserves and making predation more likely 45 . These results suggest that, while acute toxicity is not observed even at unrealistically high concentrations, further study into how $\mathrm{TiO}_{2}$ may alter $D$. magna behavior is warranted. 
453

454

455

456

457

458

459

460

461

462

463

464

465

466

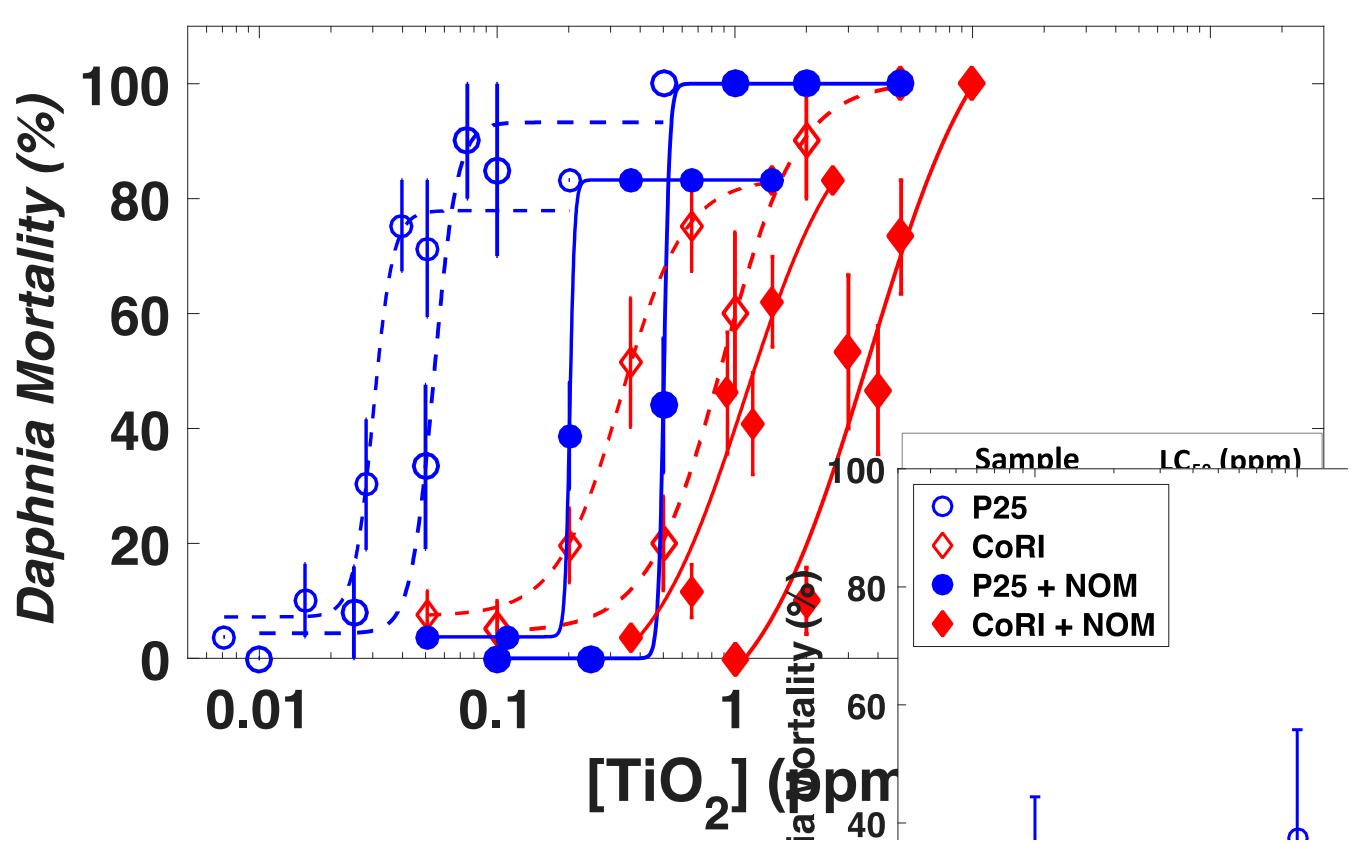

Figure 6. D. magna mortality versus $\mathrm{TiO}_{2} \mathrm{NP}$ concentration under UV light. Error bars indicate standard error. ( $\mathrm{n}=$ at least 4 runs as per OECD test protocol).

Figure 6 shows D. magna mortality versus $\mathrm{TiO}_{2}$ concentration for organisms exposed to NPs while illuminated by UV light. In comparison to visible light results, very clear dose-response curves are observed for UV exposed $\mathrm{TiO}_{2}$. Overall, D. magna toxicity under UV light followed the trend of P25 > P25 + NOM > CoRI > CoRI + NOM. These results mirror trends observed for ${ }^{*} \mathrm{OH}$ generation. The $\mathrm{LC}_{50}$ for $\mathrm{P} 25(0.05 \mathrm{ppm})$ is an order of magnitude lower than that of CoRI $(0.90 \mathrm{ppm})$ or P25 + NOM (0.50 ppm). For CoRI + NOM, the LC50 is on the order of ppm (3.67 ppm). As can be observed in all cases, P25 is more toxic than CoRI NPs. Additionally, the presence of NOM drastically reduces toxicity for a given NP type. This agrees well with the decrease in ${ }^{*} \mathrm{OH}$ observed in the presence of $\mathrm{NOM}$, both here and in previous reports where the presence of NOM has generally been shown to have a protective effect for 
organisms exposed to $\mathrm{TiO}_{2}{ }^{26}, 46-48$. The $\mathrm{LC}_{50}$ for CoRI $(0.90 \mathrm{ppm})$ is comparable to that reported by Wormington et al. for pure anatase in the absence of NOM $(0.84 \mathrm{ppm})^{26}$.

The steepness of the dose-response curve generally indicates the potency of the toxicant, suggesting that P25, even in the presence of NOM, is more "potent" than CoRI when exposed to UV light. This is especially apparent when comparing P25 + NOM and CoRI, where the range of $\mathrm{TiO}_{2}$ concentrations in which $\mathrm{P} 25+$ NOM exhibits a response but not $100 \%$ mortality is much narrower than for CoRI. This may be related to the stability of P25 + NOM in suspension, as mortality is observed at the end of $48 \mathrm{~h}$. Thus, despite the similar initial ${ }^{*} \mathrm{OH}$ generation rates $\left(3.1 \pm 0.5 \mathrm{nM} \mathrm{min}^{-1}\right.$ for $\mathrm{P} 25+\mathrm{NOM}, 1.9 \pm 0.1 \mathrm{nM} \mathrm{min}^{-1}$ for CoRI $)$, the loss of CoRI NPs from suspension over time results in a significant decrease in the cumulative ${ }^{*} \mathrm{OH}$ dose the $D$. magna are exposed to, reducing the observed mortality. In contrast, ${ }^{*} \mathrm{OH}$ generation for P25 + NOM is observed to be fairly constant over the $48 \mathrm{~h}$.

\section{It should be noted that differences in bioaccumulation and depuration between P25 and CoRI} NPs (e.g. NP shape, aggregate size) may impact the observed toxicity. While bioaccumulation was not measured in this work, no trend in mortality was observed for NPs in visible light which suggests that ${ }^{*} \mathrm{OH}$ production is the critical component in acute toxicity. However, a greater NP body burden could add an additional stress to the organism, increasing its susceptibility. Additionally, the presence of NOM, which here is linked to *OH quenching and changes in the stability of P25 aggregates, may influence the D. magna body burden compared to the same NP in the absence of NOM.

\section{Conclusions}


Concentrations of bare $\mathrm{TiO}_{2}$ NPs that are acutely toxic to D. magna under visible light conditions are found to be higher than what is realistically expected in environmental waters.

494 However, at NP concentrations in which 100\% mortality is not observed, D. magna exhibit 495 behavioral changes that suggest the potential for ecosystem impacts. UV light exposure 496 drastically increases toxicity, reducing the calculated LC50. For P25, this value (50 ppb) is on 497 the same order of magnitude as upper limit aquatic measurements and falls within 498 environmental relevance (tens of ppb) ${ }^{5,8-10}$. The presence of $10 \mathrm{ppm}$ NOM is observed to 499 reduce toxicity. In natural environments, both organic matter and sunlight are likely to be 500 present. The mitigating impact of NOM, however, is not sufficient to fully counterbalance the 501 enhanced toxicity associated with photoexcitation. Furthermore, these results also 502 underscore that not all $\mathrm{TiO}_{2}$ should be considered the same. Differences in NP stability and 503 photoreactivity are observed between the two formulations, and these factors should be 504 taken into account when assessing risk. While toxicity related solely to the particles 505 themselves are roughly the same for P25 and CoRI, P25 is shown to be more toxic under UV 506 irradiation than CoRI.

507

\section{Acknowledgements}

509 The authors acknowledge the financial support of the Canada Research Chairs program, the

510 Natural Sciences and Engineering Research Council of Canada, Environment and Climate 511 Change Canada, and the Coatings Research Institute. The authors thank G. Farley, M. Houde, 512 and M. Giraudo at Environment and Climate Change Canada for providing the source $D$. 513 magna and consultation in rearing, A. Azimzada for performing ICP-MS measurements, K. J.

514 Wilkinson for providing access to the ICP-MS, and L. M. Hernandez for performing TEM 515 imaging and XPS, and D. Liu at the Facility for Electron Microscopy Research of McGill 516 University for help in microscope operation and data collection. 


\section{References}

1. Robichaud, C. O.; Uyar, A. E.; Darby, M. R.; Zucker, L. G.; Wiesner, M. R., Estimates of upper bounds and trends in nano-TiO2 production as a basis for exposure assessment. Environ Sci Technol 2009, 43, (12), 4227-4233.

2. Hendren, C. O.; Mesnard, X.; Droge, J.; Wiesner, M. R., Estimating production data for five engineered nanomaterials as a basis for exposure assessment. Environ Sci Technol 2011, 45, (7), 2562-9.

3. Piccinno, F.; Gottschalk, F.; Seeger, S.; Nowack, B., Industrial production quantities and uses of ten engineered nanomaterials in Europe and the world. J Nanopart Res 2012, 14, (9). 4. Al-Kattan, A.; Wichser, A.; Zuin, S.; Arroyo, Y.; Golanski, L.; Ulrich, A.; Nowack, B., Behavior of TiO2 released from nano-TiO2-containing paint and comparison to pristine nano-TiO2. Environ Sci Technol 2014, 48, (12), 6710-6718.

$532 \quad 5 . \quad$ Keller, A. A.; Lazareva, A., Predicted Releases of Engineered Nanomaterials: From Global to Regional to Local. Environmental Science \& Technology Letters 2014, 1, (1), 65-70. 6. Bottero, J.-Y.; Auffan, M.; Borschnek, D.; Chaurand, P.; Labille, J.; Levard, C.; Masion, A.; Tella, M.; Rose, J.; Wiesner, M. R., Nanotechnology, global development in the frame of environmental risk forecasting. A necessity of interdisciplinary researches. Comptes Rendus Geoscience 2015, 347, (1), 35-42.

538 7. Sun, T. Y.; Mitrano, D. M.; Bornhöft, N. A.; Scheringer, M.; Hungerbühler, K.; Nowack,

539 B., Envisioning Nano release dynamics in a changing world: using dynamic probabilistic 540 modeling to assess future environmental emissions of engineered nanomaterials. Environ Sci 541 Technol 2017, 51, (5), 2854-2863.

$542 \quad$ 8. Gondikas, A. P.; Kammer, F. v. d.; Reed, R. B.; Wagner, S.; Ranville, J. F.; Hofmann, T., Release of TiO2 nanoparticles from sunscreens into surface waters: a one-year survey at the old Danube recreational Lake. Environ Sci Technol 2014, 48, (10), 5415-5422.

9. Gottschalk, F.; Sonderer, T.; Scholz, R. W.; Nowack, B., Modeled environmental concentrations of engineered nanomaterials (TiO2, $\mathrm{ZnO}, \mathrm{Ag}, \mathrm{CNT}$, fullerenes) for different regions. Environ Sci Technol 2009, 43, (24), 9216-9222. 10. Kiser, M.; Westerhoff, P.; Benn, T.; Wang, Y.; Perez-Rivera, J.; Hristovski, K., Titanium nanomaterial removal and release from wastewater treatment plants. Environ Sci Technol 2009, 43, (17), 6757-6763. environment—a review. Journal of Environmental Science and Health Part A 2009, 44, (14), 1485-1495.

554 12. Schultz, A. G.; Boyle, D.; Chamot, D.; Ong, K. J.; Wilkinson, K. J.; McGeer, J. C.; Sunahara, G.; Goss, G. G., Aquatic toxicity of manufactured nanomaterials: challenges and recommendations for future toxicity testing. Environmental Chemistry 2014, 11, (3), 207226.

13. Menard, A.; Drobne, D.; Jemec, A., Ecotoxicity of nanosized TiO2. Review of in vivo data. Environmental pollution 2011, 159, (3), 677-684.

560 14. Heinlaan, M.; Ivask, A.; Blinova, I.; Dubourguier, H.-C.; Kahru, A., Toxicity of nanosized 561 and bulk $\mathrm{ZnO}, \mathrm{CuO}$ and $\mathrm{TiO} 2$ to bacteria Vibrio fischeri and crustaceans Daphnia magna and 562 Thamnocephalus platyurus. Chemosphere 2008, 71, (7), 1308-1316.

563 15. Wiench, K.; Wohlleben, W.; Hisgen, V.; Radke, K.; Salinas, E.; Zok, S.; Landsiedel, R., 564 Acute and chronic effects of nano-and non-nano-scale $\mathrm{TiO} 2$ and $\mathrm{ZnO}$ particles on mobility 
and reproduction of the freshwater invertebrate Daphnia magna. Chemosphere 2009, 76, 566 (10), 1356-1365.

567 16. Zhu, X.; Chang, Y.; Chen, Y., Toxicity and bioaccumulation of TiO2 nanoparticle aggregates in Daphnia magna. Chemosphere 2010, 78, (3), 209-215.

17. Jacobasch, C.; Völker, C.; Giebner, S.; Völker, J.; Alsenz, H.; Potouridis, T.; Heidenreich, H.; Kayser, G.; Oehlmann, J.; Oetken, M., Long-term effects of nanoscaled titanium dioxide on the cladoceran Daphnia magna over six generations. Environmental pollution 2014, 186, 180-186.

18. Salieri, B.; Pasteris, A.; Baumann, J.; Righi, S.; Köser, J.; D’Amato, R.; Mazzesi, B.; Filser, J., Does the exposure mode to ENPs influence their toxicity to aquatic species? A case study with TiO 2 nanoparticles and Daphnia magna. Environmental Science and Pollution Research 2015, 22, (7), 5050-5058.

19. Hsiung, C.-E.; Lien, H.-L.; Galliano, A. E.; Yeh, C.-S.; Shih, Y.-h., Effects of water chemistry on the destabilization and sedimentation of commercial TiO2 nanoparticles: Role of doublelayer compression and charge neutralization. Chemosphere 2016, 151, 145-151. 20. Cupi, D.; Hartmann, N. B.; Baun, A., Influence of $\mathrm{pH}$ and media composition on suspension stability of silver, zinc oxide, and titanium dioxide nanoparticles and immobilization of Daphnia magna under guideline testing conditions. Ecotoxicology and environmental safety 2016, 127, 144-152. 21. Hoffmann, M. R.; Martin, S. T.; Choi, W. Y.; Bahnemann, D. W., Environmental Applications of Semiconductor Photocatalysis. Chem Rev 1995, 95, (1), 69-96.

22. Hoffmann, M.; Hotze, E. M.; Wiesner, M. R., Reactive oxygen species generation on nanoparticulate material. Environmental Nanotechnology. Applications and Impacts of Nanomaterials 2007, 155-203. 23. Clemente, Z.; Castro, V.; Jonsson, C.; Fraceto, L., Minimal levels of ultraviolet light enhance the toxicity of TiO 2 nanoparticles to two representative organisms of aquatic systems. J Nanopart Res 2014, 16, (8), 2559. radiation to two aquatic species: Daphnia magna and Japanese medaka. Environ Toxicol Chem 2012, 31, (7), 1621-1629.

25. Mansfield, C.; Alloy, M.; Hamilton, J.; Verbeck, G.; Newton, K.; Klaine, S.; Roberts, A.,

596

597

598

599

600

601

602

603

604

605

606

607

608

609

610

611

612 Photo-induced toxicity of titanium dioxide nanoparticles to Daphnia magna under natural sunlight. Chemosphere 2015, 120, 206-210.

26. Wormington, A. M.; Coral, J.; Alloy, M. M.; Delmarè, C. L.; Mansfield, C. M.; Klaine, S. J.; Bisesi, J. H.; Roberts, A. P., Effect of natural organic matter on the photo - induced toxicity of titanium dioxide nanoparticles. Environ Toxicol Chem 2017, 36, (6), 1661-1666.

27. Dufour, F.; Pigeot-Remy, S.; Durupthy, O.; Cassaignon, S.; Ruaux, V.; Torelli, S.; Mariey, L.; Maugé, F.; Chanéac, C., Morphological control of TiO 2 anatase nanoparticles: What is the good surface property to obtain efficient photocatalysts? Applied Catalysis B: Environmental 2015, 174, 350-360.

28. EPA, U. Methods for measuring the acute toxicity of effluents and receiving waters to freshwater and marine organisms; EPA/600/4-90: 1993.

29. Taurozzi, J.; Hackley, V.; Wiesner, M., Preparation of a nanoscale TiO2 aqueous dispersion for toxicological or environmental testing. NIST Special Publication 2012, 1200, 3. 30. Diffey, B. L., Sources and measurement of ultraviolet radiation. Methods 2002, 28, (1), 4-13.

31. Crittenden, J. C.; Trussell, R. R.; Hand, D. W.; Howe, K. J.; Tchobanoglous, G., MWH's Water Treatment: Principles and Design: Principles and Design. John Wiley \& Sons: 2012. 
32. Ishibashi, K.-i.; Fujishima, A.; Watanabe, T.; Hashimoto, K., Quantum yields of active oxidative species formed on $\mathrm{TiO} 2$ photocatalyst. Journal of Photochemistry and Photobiology A: Chemistry 2000, 134, (1), 139-142.

33. Canada, E., Biological test method: Reference method for determining acute lethality of effluents to Daphnia magna. 1990.

34. Co-operation, O. f. E.; Development, Test No. 202: Daphnia sp. Acute Immobilisation Test. OECD Publishing: 2004.

35. No, O. T., 202: Daphnia sp. acute immobilisation test. OECD guidelines for the testing of chemicals, section 2004, 2 . 36. Pikuda, O.; Xu, E. G.; Berk, D.; Tufenkji, N., Toxicity Assessments of Micro- and Nanoplastics Can Be Confounded by Preservatives in Commercial Formulations. Environmental Science \& Technology Letters 2018, 6, (1), 21-25.

37. Bhattacharjee, S., DLS and zeta potential-What they are and what they are not? Journal of Controlled Release 2016, 235, 337-351.

38. Domingos, R. F.; Baalousha, M. A.; Ju-Nam, Y.; Reid, M. M.; Tufenkji, N.; Lead, J. R.; Leppard, G. G.; Wilkinson, K. J., Characterizing manufactured nanoparticles in the environment: multimethod determination of particle sizes. Environ Sci Technol 2009, 43, (19), 7277-7284.

39. Farner Budarz, J.; Turolla, A.; Piasecki, A. F.; Bottero, J.-Y.; Antonelli, M.; Wiesner, M. $\mathrm{R}$., Influence of aqueous inorganic anions on the reactivity of nanoparticles in $\mathrm{TiO} 2$ photocatalysis. Langmuir 2017, 33, (11), 2770-2779.

40. Kosmulski, M., The significance of the difference in the point of zero charge between rutile and anatase. Adv Colloid Interfac 2002, 99, (3), 255-264.

41. Turolla, A.; Piazzoli, A.; Farner Budarz, J.; Wiesner, M. R.; Antonelli, M., Experimental measurement and modelling of reactive species generation in TiO2 nanoparticle photocatalysis. Chemical Engineering Journal 2015, 271, 260-268.

42. Latch, D. E.; McNeill, K., Microheterogeneity of singlet oxygen distributions in irradiated humic acid solutions. Science 2006, 311, (5768), 1743-1747.

43. Jassby, D.; Budarz, J. F.; Wiesner, M., Impact of Aggregate Size and Structure on the Photocatalytic Properties of TiO2 and ZnO Nanoparticles. Environ Sci Technol 2012, 46, (13), 6934-6941.

44. Hotze, E. M.; Bottero, J. Y.; Wiesner, M. R., Theoretical Framework for Nanoparticle Reactivity as a Function of Aggregation State. Langmuir 2010, 26, (13), 11170-11175.

45. Lovern, S. B.; Strickler, J. R.; Klaper, R., Behavioral and physiological changes in Daphnia magna when exposed to nanoparticle suspensions (titanium dioxide, nano-C60, and C60HxC70Hx). Environ Sci Technol 2007, 41, (12), 4465-4470.

46. Seitz, F.; Rosenfeldt, R. R.; Müller, M.; Lüderwald, S.; Schulz, R.; Bundschuh, M., Quantity and quality of natural organic matter influence the ecotoxicity of titanium dioxide nanoparticles. Nanotoxicology 2016, 10, (10), 1415-1421.

47. Yang, S. P.; Bar-Ilan, O.; Peterson, R. E.; Heideman, W.; Hamers, R. J.; Pedersen, J. A., Influence of humic acid on titanium dioxide nanoparticle toxicity to developing zebrafish. Environ Sci Technol 2013, 47, (9), 4718-4725.

48. Li, S.; Ma, H.; Wallis, L. K.; Etterson, M. A.; Riley, B.; Hoff, D. J.; Diamond, S. A., Impact of natural organic matter on particle behavior and phototoxicity of titanium dioxide 